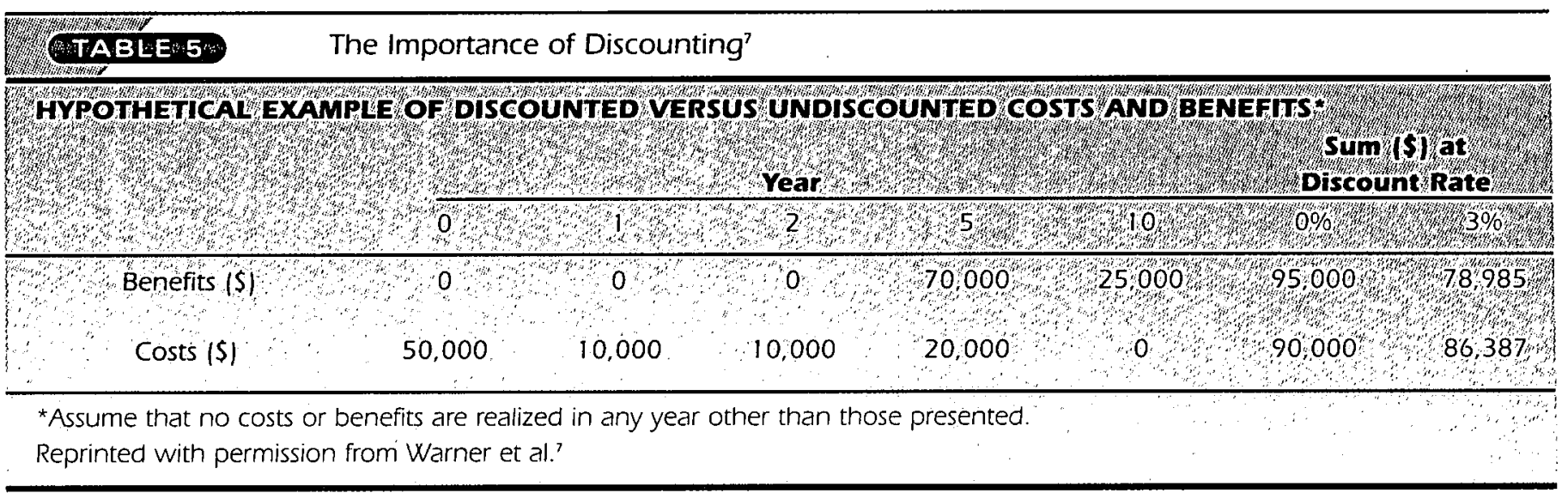

\section{Study Designs}

Four major types of study designs are used in pharmacoeconomics evaluations: clinical trials, naturalistic (or large simple) trials, models, and retrospective analyses. No study design is inherently superior to another for conducting pharmacoeconomics research. The approaches are complementary, and each has distinct advantages and disadvantages that should be considered.

\section{Clinical Trials}

The most familiar type of study to clinicians is probably the clinical trial. It typically has strong internal validity but can be of questionable relevance in the real world. Clinical trials are characterized by random assignment of subjects to study groups, a control group (often a placebo), and blinding of subjects and investigators. Clinical trials are the gold standard for demonstrating efficacy of drug therapies.

Two major advantages exist in conducting a pharmacoeconomics study using a clinical trial approach. First, the pharmacoeconomics study often can be piggybacked onto an existing clinical trial. Second, subjects are randomized to treatments, which enhances the internal validity of clinical trials (the confidence that the observed effect is due to the treatment and not some other factor). A major disadvantage is that the results are often of questionable relevance to routine practice because such controlled conditions are rarely found in clinical practice.

More specifically, clinical trials may lack an appropriate comparator. In addition, surrogate outcome markers (e.g., blood pressure) often are used to make results readily available in a timely manner. This is particularly problematic if the surrogate marker and the outcome of interest are not closely linked, which may be the case with ulcer symptoms and endoscopy-based ulcer rates.

Another limitation inherent in clinical trials is that patient compliance typically is higher than in routine care. Populations in a clinical trial are often described as "professional patients." In addition, because these patients are monitored closely, have frequent contact with providers, and receive prompting from investigators to take their medication, patients may be more compliant with the medication regimen than they would be outside a clinical trial.

Furthermore, because patient retention is an unstated goal of clinical trials, adverse effects are managed intensively, reducing the likelihood that patients will discontinue taking medication. An example of how compliance in clinical trials can be misleading appeared in recent literature. Investigators included only clinical trial data in a meta-analysis of efficacy rates of tricyclic versus selective serotonin reuptake inhibitor (SSRI) antidepressants and found no significant difference. Since then, a number of studies have demonstrated that in more routine clinical settings, substantial differences exist between the compliance rates with tricyclics and with SSRIs, particularly in the first two months of therapy, and that this compliance can affect success rates. ${ }^{9}$

\section{Naturalistic Trials}

The alternative to the clinical trial is the naturalistic, or large simple, trial. Its goal is to eliminate most of the disadvantages of a clinical trial while retaining randomization and a control group. Typically, naturalistic trials are conducted in the Phase IV Postmarketing stage. Settings and providers are more representative of routine clinical practice than those in a clinical trial. Patients and providers are not blinded to the treatment received. Inclusion and exclusion criteria are less extensive. In addition, the drug being evaluated is compared with standard care rather than a placebo. The antidepressant trial discussed previously is an example of a naturalistic trial."

The primary advantage of naturalistic trials is that they more accurately reflect routine clinical care. However, naturalistic trials typically can be even more expensive than clinical trials and, in many instances, are not a practical alternative. Furthermore, a naturalistic trial may not be as representative as the investigators claim. Decision makers should read the methodology carefully and decide whether the trial accurately mirrors clinical practice 


\section{TAB E E 6}

$\% \%$ \%का

\section{When to use?}

Clinical trial does not capture economic data

Measures clinical intermediaries only

Compares treatment with placebo

Want to translate clinical trial data into that which better reflects routine clinical care

\section{Models}

Models are commonly used in pharmacoeconomics research and comprise an increasing portion of published studies. A model is defined as a systematic approach to decision making under conditions of uncertainty. A model is used when direct observation is not available and prospective data cannot be collected. A model does not banish uncertainty but makes the uncertainty explicit by including junctures at which a decision must be made or the likelihood of an outcome predicted. In pharmacoeconomics, investigators use two major types of models -decision-analytical and Markov. The latter are sophisticated and represent a minority of the models in the literature; decision-analytical models are used more often.

Figure 5 is an example of a decision-analytical model of ulcer pharmacotherapy. Each square box is called a decision node, where a choice is made about which treatment alternative to use. Two branches are shown here, but other branches could be added at this decision node, such as the dual-based or triplebased proton pump inhibitor regimens.

Once a triple therapy has been selected, the clinical course can take more than one direction. The circle on the model is called a chance node or a probability node. At this juncture, a probability that the Helicobacter pylori bacteria will be eradicated can be assigned to one branch and a probability that it will not to the other. The assigned probabilities are based on the literature and should total $100 \%$. The two branches at the next chance node represent the probability that the ulcer will recur and the probability that it will not. Even if the ulcer is eradicated, the probability exists that the patient will have a recurrence; this probability is much lower than if the ulcer had not been eradicated, but it exists nevertheless. Beyond those chance nodes- "ulcer" and "no ulcer recurrence"-a chance exists that an outpatient visit will occur, as well as a chance that the patient will be hospitalized.

The reader should then critically analyze a model to determine whether the model has been constructed as simply as possible, yet still represents clinical reality. The model shown here is reasonable because it captures several important elements in the course of treating a patient with an ulcer.

Table 6 details the situations in which models are useful. For example, if no pharmacoeconomics data were collected during a clinical trial but the investigators want to perform a pharmacoeconomics analysis, a model can be developed that incorporates the efficacy data from the clinical trial and cost data from other sources. If the clinical study has measured only intermediary or surrogate measures, the investigators can use a model to extrapolate those results to a lifetime.

Assume one clinical trial has compared drug $X$ with placebo and another compared drug $\mathrm{Y}$ with placebo. The investigator later decides to conduct a head-to-head comparison of the two drugs. The results from the two clinical trials may be used to model the economic impact of selecting drug $\mathrm{X}$ versus drug $\mathrm{Y}$. In addition, if the investigator has clinical trial data and wants to construct a model that more closely mirrors routine clinical care, data from medical claims databases and the literature can be used.

For example, to compare the cost of outpatient treatment of depression with tricyclics versus SSRIs, the investigator knows

Outpatient

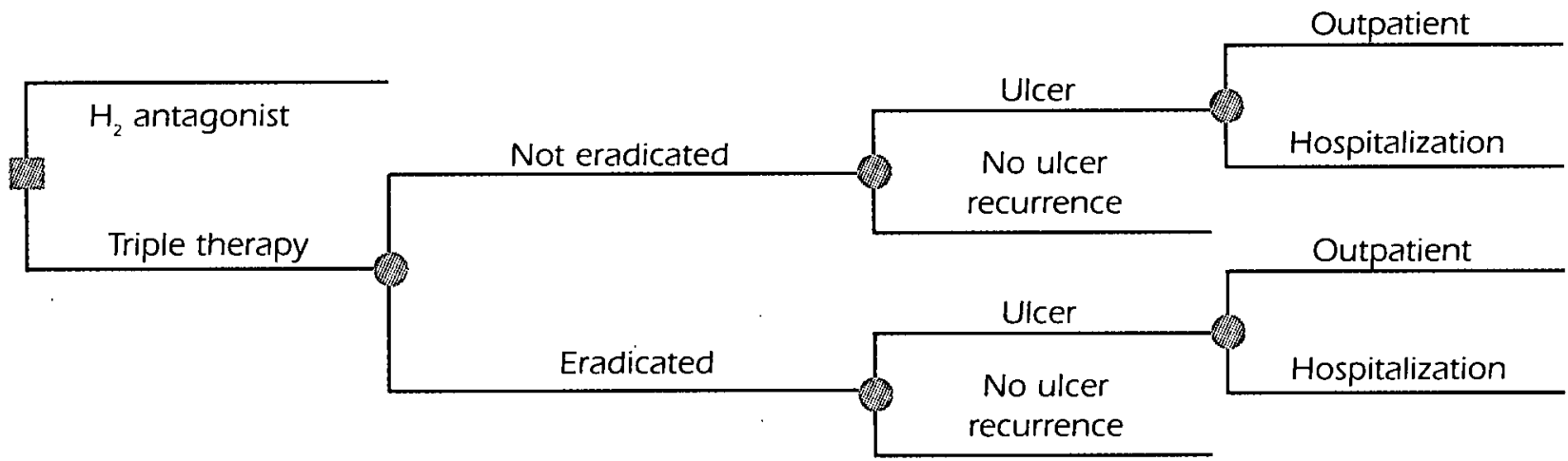




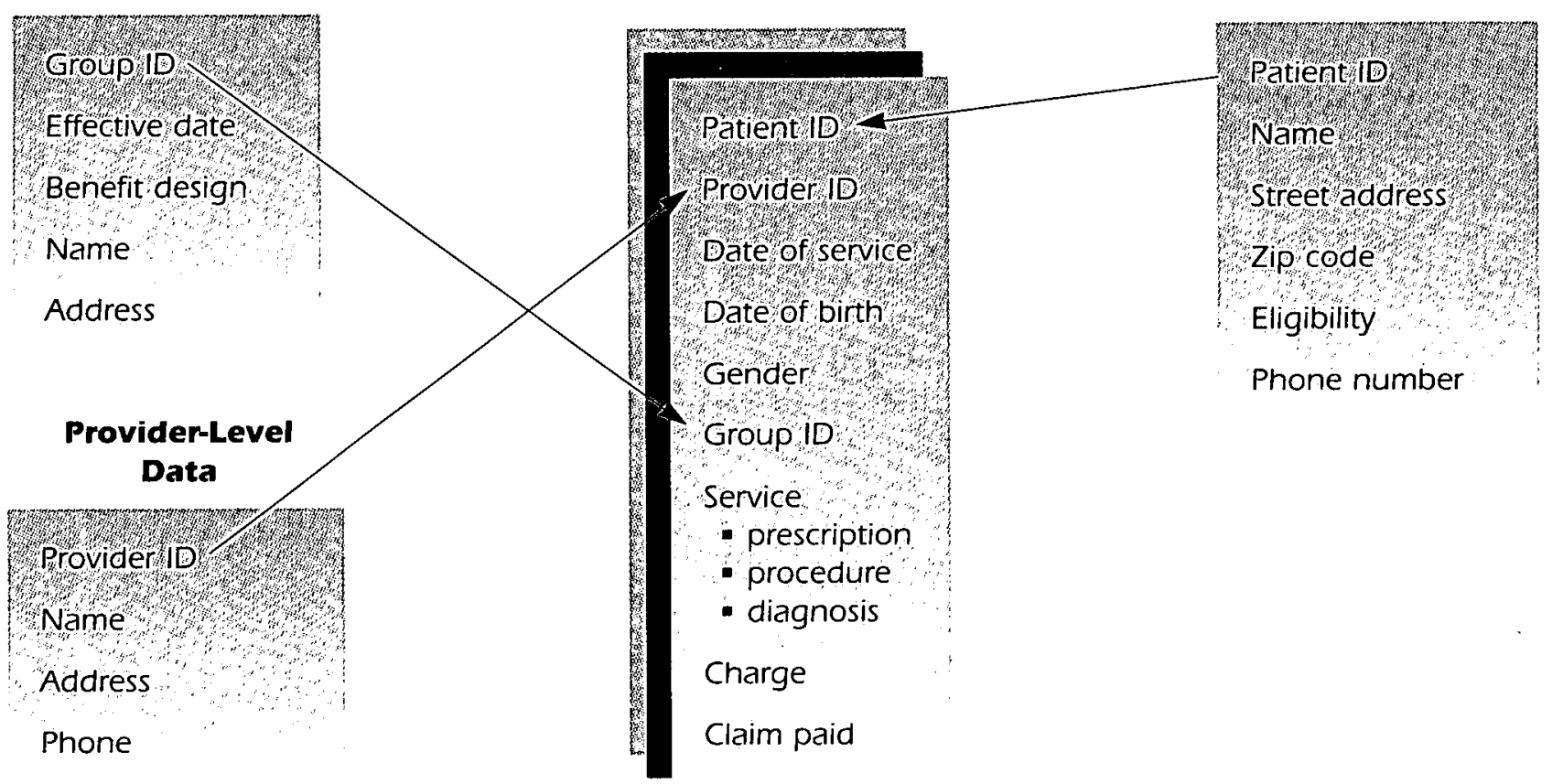

from the literature that compliance is X for the SSRIs and Y for the tricyclics; the probabilities can be input into a model. The investigator might want to know other probabilities as well, such as the percentage of patients that will require a physician visit to manage side effects for each drug or the percentage who attempt suicide while taking each drug

Like the other approaches, models have limitations. The structure and logic of a model may not reflect clinical practice. Is the comparator the most relevant alternative? For example, the model discussed previously compares an $\mathrm{H}_{2}$ antagonist with triple therapy for ulcer treatment, but an $\mathrm{H}_{2}$ antagonist is no longer the standard of care. Therefore, the model is of limited use.

Models sometimes are viewed as "black boxes." When a pharmaceutical company representative brings a laptop computer model comparing one of their products with a competitor's product, information about the derivation of probabilities and other estimates may not be clear. Fortunately, an increasing number of companies are making their models more transparent. In some cases, the user simply can push a button to find the source of the probability estimates and/or enter a different set of probabilities and view the changes that result in the model.

An additional limitation of models is the reliability of the probability estimates. For example, one model assumed that
$95 \%$ of patients with ulcer symptoms will undergo endoscopy, which is not a reasonable assumption. Estimates of cost also need to be viewed critically.

\section{Retrospective Analyses}

The vast majority of retrospective analyses are based on information from claims databases (see Figure 6). Claims databases have several advantages. They reflect routine clinical care, are comprehensive and provide large sample sizes. The investigator can avoid some ethical concerns because randomization is not an issue in a retrospective analysis. Certain biases can be avoided-for example, patients and physicians probably will not change their behavior based on the expectation that an investigator will conduct a claims database study. In a retrospective analysis, the follow-up periods are longer than those found in most clinical trials. Typically, an investigator using a claims database can follow a sample population for one to two years. After that, the sample characteristics may change because people leave the plan. Finally, compared with clinical trials, studies of claims databases are relatively inexpensive, and the research can be done in a timely fashion.

Despite these advantages, retrospective analyses have some major limitations (see Table 7, p. 12). The biggest disadvantage is the problem of internal validity. If a study finds that drug $A$ is associated with higher overall medical costs than drug $B$, one 


TABYE 7 . Claims Databases

\section{Limitations}

- Questionable internal validity

-Channeling bias

- Diagnostic information

- Undercoding, overcoding, coding changes

- Patient identification, costs

Missing information

$\rightarrow$ Reimbursed services only, claim lag

Multiple indieations

Datàstructure:

Eligibility, capitation family linkage

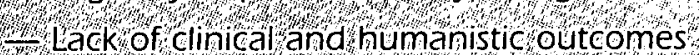

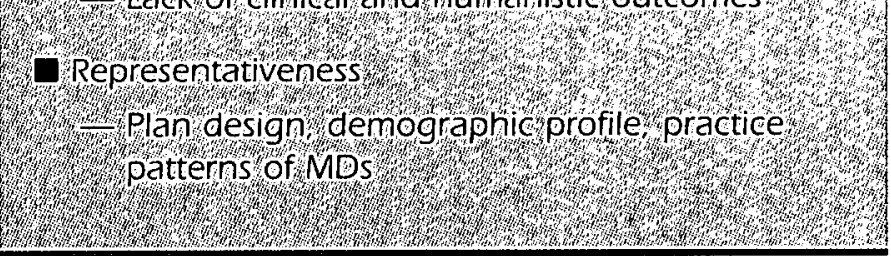

\begin{tabular}{|c|c|}
\hline GIABLE & $\begin{array}{l}\text { Critical Assessment of } \\
\text { Economic Evaluations }\end{array}$ \\
\hline
\end{tabular}

Well-defined question

- Description of competing alternatives

- Effectiveness established

- Relevant costs and consequences identified

- Costs and consequences measured accurately

Costs and consequences valued credibly

Costs and consequences adjusted for differential timing

sensitivity analysis orformed

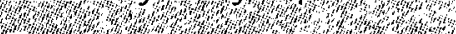

presentation and discussion of study results

include all issues of concern to users

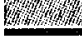

cannot necessarily conclude that the use of $\operatorname{drug} \mathrm{A}$ is causing the high costs, because the patients were not randomized to treatment arms.

Consider the following example: A pharmacist may be asked to use claims data to determine the cost-effectiveness of prescribing inhaled steroids to all patients with moderate-to-severe asthma. The pharmacist retrieves utilization and cost data for all asthma patients who consistently used inhaled steroids and all who took beta-agonists over one year. The data show that costs are significantly higher for patients taking inhaled steroids. Should the pharmacist conclude that steroids are not cost-effective? No, asthma patients who use inhaled steroids usually have more severe asthma than patients taking only beta-agonists. Unless the pharmacist can control for the severity of asthma, which probably cannot be done using a claims database, a retrospective analysis of this type will be inappropriate and potentially misleading.

The term for this methodologic problem is channeling biaspatients receive certain products for specific reasons. If the researcher cannot identify and control for this bias, inferring cause-and-effect relationships is inappropriate. Yet this is a common error in the pharmacoeconomics literature. Databases can easily be used to demonstrate correlations, but causation cannot be assumed.

Problems with diagnostic information and missing information are also disadvantages of using claims databases. For example, a diagnosis of depression may be undercoded for many reasons, such as the patient's or physician's fear that health insurance will be denied in the future. In addition, using a claims database to evaluate the impact of a drug with multiple indications is particularly difficult. For example, because beta-blockers are prescribed for patients with cardiovascular disease, migraine and other conditions, a diagnosis cannot be inferred solely from the fact that a patient has taken this type of medication.

Eligibility information also is critical. Only patients who were eligible to receive health insurance benefits during the entire study period should be included. Otherwise, it will be impossible to determine if a patient was not hospitalized because he or she did not go to the hospital or because the patient was ineligible for hospital coverage at that time.

Clinical outcomes may be limited when using claims databases, and humanistic outcomes are nonexistent in the databases. Although databases are typically more representative of practice than are clinical trials, plan design, demographic profile and practice patterns still may influence whether a claims database study is generalizable to other settings.

\section{Evaluating Pharmacoeconomics Studies}

\section{Using a Checklist}

When analyzing a study, a checklist of important elements that each pharmacoeconomics study should include is a useful tool (see Table 8). The first item on the list is the study objective. Skim the abstract to determine whether the objective is clear and whether the research question is relevant. Does this study apply to an area of interest or an upcoming question? Is the approach used to research the question appropriate?

The author of a published study should state explicitly the alternative therapies being compared, even if the alternative is 